\title{
Long non-coding RNA UCA1a(CUDR) promotes proliferation and tumorigenesis of bladder cancer
}

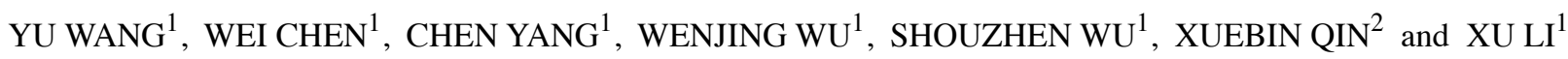 \\ ${ }^{1}$ Center for Molecular Medicine, The First Affiliated Hospital, School of Medicine, Xi'an Jiaotong University, Xi'an, P.R. China; \\ ${ }^{2}$ Department of Medicine, Brigham and Women's Hospital, Harvard Medical School, Boston, MA, USA
}

Received January 12, 2012; Accepted March 7, 2012

DOI: $10.3892 /$ ijo.2012.1443

\begin{abstract}
Our previous studies identified UCA1 as a novel biomarker for bladder cancer and detected three variant transcripts of UCA1 in the human bladder TCC cell line BLZ-211 using northern blot analysis. One $(1.4 \mathrm{~kb})$ of the three transcripts has been shown to play a pivotal role in bladder cancer progression and embryonic development. In this study, we cloned a second transcript $(2.2 \mathrm{~kb})$, designated UCAla, which was identical to previously reported cancer upregulated drug resistant gene (CUDR). Sequence comparison of UCA1 (1.4 kb transcript) and UCA1a(CUDR) cDNA revealed a 1,265 bp common region. Previous studies have demonstrated that CUDR is upregulated in various human tumors, including colon, cervical and lung cancer. However, the exact role of UCA1a(CUDR) in bladder cancer has not yet been reported. In this study, RT-PCR analysis indicated that UCA1a(CUDR) was also an embryonic development and bladder cancer-associated RNA. Overexpression of UCA1a(CUDR) significantly enhanced proliferation, migration and invasion of the bladder cancer cell line UM-UC-2. Moreover, microarray analysis demonstrated that overexpression of UCA1a(CUDR) was associated with signaling pathways regulating cell apoptosis and tumorigenesis. Furthermore, overexpression of UCA1a(CUDR) could antagonize cell apoptosis induced by cisplatin and promote the tumorigenicity of UM-UC-2 cells in vivo. Taken together, our data strongly suggest that similar to the $1.4 \mathrm{~kb}$ transcript of UCA1, UCA1a(CUDR) may also play an important role in the growth and tumorigenesis of human bladder cancer, and their common region may be critical for biological activity, thereby indicating that their common region may serve as a new therapeutic target for bladder cancer.
\end{abstract}

Correspondence to: $\mathrm{Dr} \mathrm{Xu} \mathrm{Li}$, Center for Molecular Medicine, The First Affiliated Hospital, School of Medicine, Xi'an Jiaotong University, Xi'an, P.R. China

E-mail: lixu1956@gmail.com

Key words: long non-coding RNA, UCA1, UCA1a(CUDR), proliferation, bladder cancer

\section{Introduction}

In recent years, a growing number of long non-coding RNAs (lncRNAs) have been identified with low coding potential, possessing only short open reading frames (less than 200 nucleotides), but potentially coding small peptides with 50-70 amino acids (1). Emerging data have demonstrated that lncRNAs are crucial for the regulation of chromatin structure, gene expression, and translational control (2-4). Furthermore, such lncRNAs may be involved in pathogenesis of disease, most notably in cancer development (5). There is evidence to suggest that lncRNAs may regulate key cancer pathways at the transcriptional, posttranscriptional, and epigenetic levels (6). However, few studies are focusing on the involvement of IncRNAs in bladder cancer progression.

Using the subtractive suppression hybridization (SSH) technique, we previously reported an expressed sequence tag (EST) (GenBank Accession no. DR159656) isolated from two bladder transitional cell carcinoma (TCC) cell lines, BLS-211 and BLZ-211 (7). Based on this EST, we identified an IncRNA named UCA1 $(1.4 \mathrm{~kb})$ from BLZ-211 cells using the rapid amplification of cDNA ends (RACE) (8). Northern blotting indicated that UCA1 was composed of three major transcripts $(1.4,2.2$ and $2.7 \mathrm{~kb}$ ) in BLZ-211 cells, and the $1.4 \mathrm{~kb}$ transcript was the most abundant (8). Previous studies have demonstrated that the $1.4 \mathrm{~kb}$ transcript may be a novel biomarker for bladder cancer (9) and may play a pivotal role in bladder cancer progression and embryonic development (8). However, whether other transcripts can play same roles in bladder cancer, is still unknown.

Using in silico cloning, 5'- and 3'-RACE, we successfully identified the full-length cDNA sequence of another transcript of UCA1 gene, designated as UCA1a ( $2.2 \mathrm{~kb})$, which was identical to previously reported cancer upregulated drug resistant (CUDR) gene. Interestingly, sequence comparison of the UCA1 and UCA1a(CUDR) cDNA revealed a 1,265 bp common region. CUDR was believed to exert its function as an IncRNA in the regulation of drug resistance and cellular transformation (10). In addition, CUDR was upregulated in various tumor tissues, including colon, cervix, and lung cancer (10). However, the role of UCAla(CUDR) in bladder cancer has not yet been reported. In the present study, we aimed at unveiling the function of UCA1a(CUDR) in the regulation of bladder cancer development, growth, and invasion. Our results supported the hypothesis that 
Table I. Primer sequences used in this study.

Gene name

Primer sequence

\section{5'-RACE GSP1 \\ 3'-RACE GSP2 \\ 5 '-end primer \\ 3 '-end primer \\ Fas (NM_000043)}

ATM (NM_000051)

PDGFB (NM_002608)

GAPDH (NM_004360)

\author{
5'-CCAGGGACTGCGTGGACACCTTAAAG-3' \\ 5'-GAGCCAAGAAGTCTGGAGCAGCCG-3' \\ 5'-GCTGACTCTCTTTTCAGACTCAGCC-3' \\ 5'-TTTCGTCTGACTCTTTTAGGAAGATTTC-3' \\ 5'-CCCAGAATACCAAGTGCAGA-3' \\ 5'-CTGGTGAGTGTGCATTCCTT-3' \\ 5'-CCGTGATGACCTGAGACAAG-3' \\ 5'-GAACACCACTTCGCTGAGAG-3' \\ 5'-CAAACTCGGGTGACCATTC-3' \\ 5'-CTTCAGTGCCGTCTTGTCAT-3' \\ 5'-TGGATGGCCCCTCCGGGAAA-3' \\ 5'-ACCTTGCCCACAGCCTTGGC-3'
}

similar to UCA1, UCA1a(CUDR) might also play a pivotal role in bladder cancer progression, and their common region may be critical for the biological activity.

\section{Materials and methods}

In silico cloning. Using an EST fragment (GenBank Accession no. DR159656) as an information probe, we searched the EST database in GenBank. A total of 95 ESTs were obtained by sequence walking. These ESTs were assembled into clusters of overlapping sequences using the CAP3 program (11) with criteria of at least 30 bases overlap and $95 \%$ identity between one end of a read and the one end of other reads. An EST contig with the length of 2,352 bp was obtained.

Cell culture and tissues collection. Human bladder cancer cell lines BLZ-211, 5637 and UM-UC-2 were maintained in RPMI-1640 medium (Gibco-BRL, Gaithersburg, MD, USA) supplemented with $10 \%(\mathrm{v} / \mathrm{v})$ bovine calf serum at $37^{\circ} \mathrm{C}$ in a humidified atmosphere with $5 \% \mathrm{CO}_{2}$. All tissue samples were obtained during surgical resection from the First Affiliated Hospital, School of Medicine of Xi'an Jiaotong University. Tissue samples were collected with written consent from patients, which were approved by the Hospital Ethics Committees. All samples were pathologically confirmed.

5'- and 3'-RACE. The full-length of UCAla gene was obtained using RACE with the SMART RACE cDNA Amplification kit (Clontech, Mountain View, CA) by following the manufacturer's instructions. For template, $1 \mu \mathrm{g}$ of total RNA obtained from BLZ-211 cells was used. Specific 5'- and 3'-cDNA fragments were amplified with the universal primer mix provided by kit and gene specific primers (GSPs) with the advantage 2 PCR polymerase mix (Clontech). The PCR products were cloned into pGEM-T Easy vector (Promega, Madison, WI, USA), and then sequenced. Based on the sequence of 5'- and 3'-cDNA fragments, the 5'- and 3'-end primers were designed to clone the full-length cDNA of UCA1a. All primer sequences are listed in Table I.
Sequence analysis of UCA Ia. BLAT was used to map the cDNA to chromosome. BLAST was applied to align the sequences.

Semiquantitative reverse transcription-polymerase chain reaction (RT-PCR). RNAs from cells and tissues were extracted and reverse-transcribed with ImProm II reverse transcriptase (Promega). Primer sequences for 25 cycles of PCR amplification were as follows: UCA1a(CUDR) (forward: 5'-GCACCCT AGACCCGAAA-3'; reverse: 5'-GCCACCTGGACGGATAT-3'), UCA1 (forward: 5'-CTCTCCATTGGGTTCACCATTC-3', reverse: 5'-GCGGCAGGTCTTAAGAGATGAG-3'), 18S (forward: 5'-CAGCCACCCGAGATTGAGCA-3', reverse: 5'-TA GTAGCGACGGGCGGTGTG-3'). Annealing temperature was at $54^{\circ} \mathrm{C}$.

Stable transfection of UCAI and UCA Ia(CUDR) cDNA. The full-length UCA1a(CUDR) plasmid was digested with BamHI and EcoRI restriction enzymes and subcloned into the pcDNA3.1 mammalian expression vector (Invitrogen). Thereafter, UM-UC-2 cells were, respectively, transfected with the pcDNA/UCA1a(CUDR) constructs and the prepared pcDNA/UCA1 constructs (8) using FuGENE HD Transfection Reagent (Roche, Mannheim, Germany) according to the manufacturer's instructions. The pcDNA3.1 empty vector was used as control. Colonies were then trypsinized, and the positive clone was identified by RT-PCR for UCA1, UCA1a(CUDR) and neo gene expression. The primers of neo were as follows: forward, 5'-ACAAGATGGATTGCACGCAGG-3'; reverse, 5'-TTCTCGGCAGGAGCAAGGTGA-3'. Annealing temperature was at $54^{\circ} \mathrm{C}$.

Cell proliferation, colony formation. Cell proliferation was assessed by using the 3-[4,5-dimethylthiazol-2-yl]-2,5-diphenyltetrazolium bromide (MTT) (Amresco, Solon, OH, USA) assay. Five thousand cells per well were seeded in 96-well plates in culture medium containing $10 \%$ fetal bovine serum (FBS), and then routinely cultured for 6 days. Cells were incubated in $20 \mu \mathrm{l}$ of a $5 \mathrm{mg} / \mathrm{ml}$ solution of MTT for $4 \mathrm{~h}$ at $37^{\circ} \mathrm{C}$ and lysed in $150 \mu \mathrm{l}$ of dimethyl sulfoxide (DMSO) for $10 \mathrm{~min}$ at room temperature. 
The absorbance in each well was measured at $490 \mathrm{~nm}$ by a microplate reader.

For colony formation assay, cells were harvested after trypsinization, and resuspended in the complete medium. Single cell suspensions were plated into 6-well plates at the clonal density of 200 cells per well. Plates were then incubated for 2 weeks in a $37^{\circ} \mathrm{C}$ incubator. The number of colonies was counted after staining with Giemsa for $30 \mathrm{~min}$.

Cell motility and invasion assay. Cell migration or invasion assay was performed using Millicell chambers. Inserts containing $8-\mu \mathrm{m}$-pore size filters were either non-coated for migration or coated with Matrigel (Becton-Dickinson Labware, Bedford, MA) for invasion assay. A total of $10^{5}$ cells in $100 \mu \mathrm{l}$ of serum-free medium was added to each. Twenty-four hours after incubation at $37^{\circ} \mathrm{C}$, cells that had migrated through the filter pores were fixed with methanol, stained with Giemsa, and counted in 10 random fields (x200).

Microarray analysis. Total RNAs were isolated from UM-UC-2 cells that stable transfected by pcDNA/UCA1, pcDNA/UCA1a (CUDR) or pcDNA3.1 plasmids. RNAs were then subjected to reverse transcription, labeled, and hybridized to the Whole Human Genome Oligo Microarray (KangChen Bio-tech, Shanghai, China), which contained 41,000+ unique human genes and transcripts having public domain annotation. Average linkage hierarchical clustering was carried out on genes filtered by detection flags and signal intensity. Pathway analysis was also utilized to identify the significant canonical pathways.

Quantitative real-time-PCR. According to the results of microarray analysis, genes with more than 2-fold upregulation or downregulation [pcDNA/UCA1 vs pcDNA3.1 or pcDNA/ UCA1a(CUDR) vs pcDNA3.1] were further validated by qPCR. The reactions were carried out in an ABI PRISM 7300 Sequence Detection System (Applied Biosystems, Foster City, CA, USA) with the SYBR ${ }^{\circledR}$ Premix Ex Taq ${ }^{\text {TM }}$ II kit (Takara Biotechnology, Dalian, P.R. China) and using the following cycling parameters: initial denaturation at $95^{\circ} \mathrm{C}$ for $10 \mathrm{sec}$, followed by 40 cycles of $95^{\circ} \mathrm{C}$ for $5 \mathrm{sec}, 59^{\circ} \mathrm{C}$ for $15 \mathrm{sec}$ and $72^{\circ} \mathrm{C}$ for $31 \mathrm{sec}$. Fluorescence intensity of the amplified products was measured at the end of each PCR cycle and the results were normalized to internal control glyceraldehyde-3-phosphate dehydrogenase (GAPDH) mRNA. All primer sequences are listed in Table I.

Apoptosis analysis. Cells $\left(6 \times 10^{5}\right)$ transfected with pcDNA/ UCA1, pcDNA/UCA1a(CUDR), or pcDNA3.1 empty vector cells were seeded separately in 6-well plates for $24 \mathrm{~h}$. Cells were then treated with cisplatin $(20 \mu \mathrm{M})$ for $48 \mathrm{~h}$. Afterwards, cells were trypsinized, washed twice with PBS, and resuspended in PBS. The cells were then stained with Annexin V/propidium iodide (PI) for $15 \mathrm{~min}$ in the dark at room temperature. Cell populations were analyzed by flow cytometer.

Tumorigenesis assay. The athymic nude mice (4-weeks-old) for in vivo tumorigenicity assay were purchased from Xi'an Jiaotong University (Xi'an, China). One week after acclimatization, the mice were randomly divided into three groups ( $\mathrm{n}=4 \mathrm{mice} / \mathrm{group}$ ). Cells transfected with pcDNA/UCA1, pcDNA/UCA1a(CUDR), or pcDNA3.1 empty vector were harvested and resuspended in serum-free RPMI-1640 medium. Cells $\left(7 \times 10^{6}\right)$ in $100 \mu \mathrm{l}$ of serum-free RPMI-1640 medium from different transfections were injected subcutaneously into the left flanks of each mouse. All mice were euthanized at 5 weeks after the initiation of the experiment, and tumors were excised for measurement of tumor weight. All animal experimental procedures were approved by the Institutional Animal Care and Use Committee of Xi'an Jiaotong University, and all mice were treated according to the Animal Care and Use Guidelines approved by the National Institutes of Health (Bethesda, MD, USA).

Statistical analysis. All of the in vitro experiments were performed in triplicate at least. The data are presented as the mean values \pm SD. Comparisons between groups were evaluated by a two-tailed Student's t-test. $\mathrm{p}<0.05$ was considered significant.

\section{Results}

Identification and sequence analysis of another transcript of UCAl gene, UCA la. We identified and cloned another transcript of UCA1 gene in this study. By searching the NCBI database, we retrieved a total of 95 ESTs and a 2,352 bp contig was obtained by assembling these EST sequences by CAP3 program. Based on this new contig, we designed 5'- and 3'-RACE GSPs (Table I). For 5'-RACE products, the amplified products were sequenced. For 3'-RACE products, the longest amplified products were sequenced in order to obtain the maximum amount of sequence at the 3'-end. With end-to-end PCR, the 5'- and 3'-end primers amplified a single cDNA (Fig. 1A). Thus, the cloned full-length cDNA of UCA1a was 2,202 bp (Fig. 1B, GenBank Accession no. GU357550), which was consistent with the result of northern hybridization (8).

The identity of UCAla was revealed by mapping with the BLAST search program from NCBI. The UCA1a cDNA was found to have $100 \%$ identity with the human part of the DNA sequence of the chromosome 19 cosmid (GenBank Accession no. AC004510.1). As shown in Fig. 1C, the full-length of UCA1a cDNA was mapped to 19p13.12 with three exons. Moreover, the UCA1a cDNA (nucleotides 1-2202) had 99\% identity with human CUDR gene (nucleotides 33-2245), which was also $2.2 \mathrm{~kb}$ long and mapped to human chromosome 19p13.1 [GenBank Accession no. GP468879.1; (10)]. Therefore, sequence analysis of UCA1a gene revealed that it was identical to previously reported CUDR gene. Interestingly, sequence comparison of the UCA1 and UCA1a(CUDR) cDNA revealed unique 5'- and 3'-ends and a 1,265 bp common region (red region, Fig. 1D).

The expression patterns of UCAla $(C U D R)$ in cells and tissues. Our previous results have demonstrated that UCA1 $(1.4 \mathrm{~kb})$ was an embryonic development and bladder cancer-associated RNA (8). In addition, there was evidence to suggest that CUDR was only expressed in human placenta but not in other normal tissues; CUDR was upregulated in human colon, cervix, and lung cancer (10). These results guided us to investigate the role of UCA1a(CUDR) in embryonic development and bladder cancer progression. Therefore, the expression patterns of UCA1a(CUDR) in various phase embryo tissues, adult tissues, bladder cancer cell lines and tissues were examined using semiquantitative PCR. Consistent to that of UCA1 (8), UCA1a(CUDR) was also expressed in all stages during embry- 
A
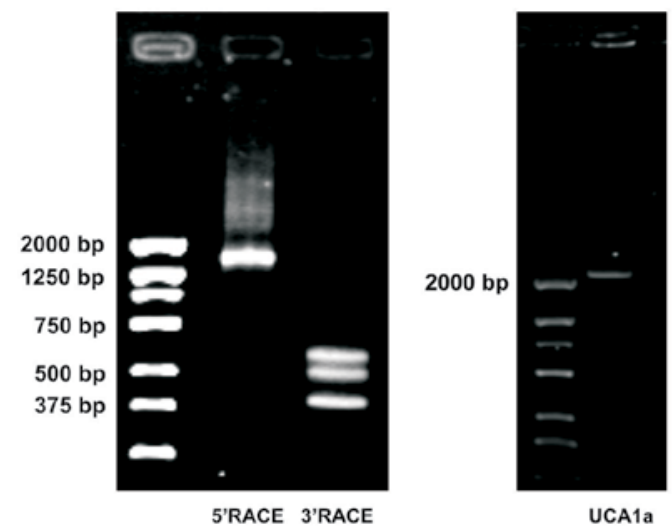

\section{Genome}

gi|224589810:15939757-15947134 Homo sapiens chromosome 19 GRCh37.p2 primary reference assembly

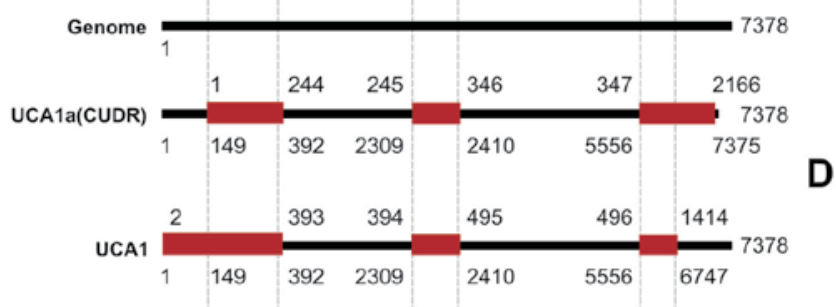

B

GCTGACTCTC TITTCAGACT CAGCCCACTT GCACOCAAGT GAATTAACAG OCTT GTTGCT CACACAAAGC CT GTTTAGGT GGTCTTCTAT ACGGACATGC TTGACACTT G GT GCCAAAAT CTGGBOCAGG GGGACTOCTT CGT GAGACCG GCOCOCTGTC CTGGCCCTCA TTOCGT GAAG AGATCCACCT GCСACCTCGS GTCCTCAGAC CAOCOCAAGO AACATCT CAC CAATTT CAAA TOGOATCTCC TCOOCTTAGI OOCTCAACAC TCAT $\cong$ CTGCC COATCOCCTC ACAACCOCC TGEACCATCA CAGATGCCGA GCITCGGGTA ACTCTIACGG TGCAGGATTC OCAGOCATAT TGEACCATCA CAGATGOCGA GCTTCGGGTA ACTCTIACGG TGCAGGATTC OCAGOCATAT GAGACACCC TAGCTGGACG ATCAGTCCIT GICAAAGTC TGAOCOCTCA AACTCTACAG

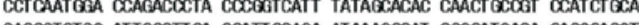
GACOCTCTCC AITGGGTTCA CCATTCCAGA ATAAAGOCAT GCCCATCAGA CAOCCA@CT GATCT CTOCT CTTCCT $\cong$ CTG GAAGCCACAA GATTAGOCOO AGAOCOGATC AGACAAACAA CCTACAACCC TIAAGCTCCT GGCAGCGOCC AGOCAAGG $\cong$ ATGCTTCCAT $Ф C A A C A C T \subseteq C$

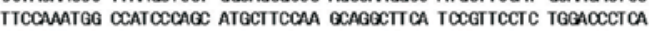
TCTCTTAAGA OCTGOCGCCT ATAAAAAGCA TTATATCTTG AGAOCCTATC CTCT AAAATT TTTTCCACAC OCAAAACAAA AAATCTCTGG GTCAAAAGTC TAAAACGCTT AGGCTGGCAA CCATCAGATC CITOCCCATG GTGTCCTCAA GCCTACTCTC ATGAAATGGA CAACAGTACA COCATATOBO GCCAGTTCCA CATATTTOOC AAOCAOACCA OCATCCAOGA CAACACAAAO TATOTTOTIT GITOTTABAB COCTTMBGAC ATTTCACTCT TTBOCABOCT CAGCTT CAGCOCACA AQATTATTTT CCTTATTATC TCTTCTOCAT AMGATCTOCA ATCACAACTA

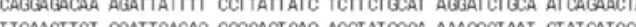
TTGAACTTCT OCATTCAGAC CGCCACF CAC ACCT RT GGGA AAAGGGT AAT GTATCATCGS CTTAGCAACA GGGAATACTA TTCGTAT GAT GGAAAATGGO GACAAAAGGC TTTGGTACAT AAAACATTAT TCCTTOCTFO COCTAAAAT TCATCOCCAC CTACATTAAA OCTAATATGC CTGATTACTG TITTTAGAGA ACTTATTTTA TTAGGGCAGT TCCAAGCTCA AAAATADGCT AACTGGCACC TTGTTAGCTA CATAAAAATG CAOCCTAGAC CCGAAACTTA CTAGACTCAT TATAAAATTT TCTTTAAGGT GTCCACBCAG TCOCTQGTCA CACTT $Q A A G C$ AGTCOGGAGA AATATCAGCC CTACOCCAGT AATOCCCAGA AGCAACTTAC ACTTTITTTT AATCTTTTO TACAACTTCA TATTTTATAA ATAAAAAAGAC AAAAATGTCA GOCCT GT GAG CT GAAGCTTA GCCATTOTAA OCOCTGTQAC CTOCACATAT OCGT OCAGGT GOCCTOCAGO AOOCAACAAD TCTGBAGCAG OCCAAAAACC ACAAACAAGT GAAACAGCCA GTTTCTGOCT TAACTAATTA ACCCACCTTA DGACATTCCA CCATTAT GAC TTGTOCACCA TTATGACTT G TTOCTGOCCT GOCOCAACT G ATCAATCAAC CCT GT GACAT TCTTCTOCTO GACAATGAGT OCCATCATCT CTCCACCATO CACCTIGTGA COCCCTCCTC TOCT GAGGAT AACCACCTTT AACT GTAACT TTCOACOCOT ACOCAAOCO TATAAAOCTO OCOOTCTCGT ATCTCOCTTC ACTOAOTCTO

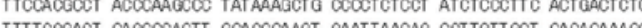
TITTCGCACT CAQCCCACTT GCACCCAAOT GAATTAACAO CCTTGTTOCT CACACAAAGC

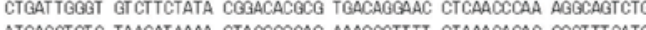
ATGAGGT GTC TAAGAT AAAA GTAGCGSCAC AaAOGCTTTT GTAAACAGGG GCGT TT CATG

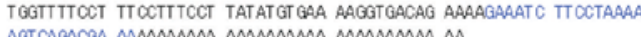
AGT CAGACGA AAAAAAAAAAA AAAAAAAAAAA AAAAAAAAAAA AA

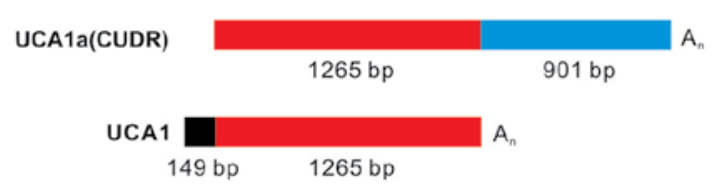

Figure 1. Identification of UCA1a full-length cDNA. (A), 5'- and 3'-ends of UCA1a were amplified from BLZ-211 cells using RACE technique (left panel). The full-length cDNA of UCA1a was amplified from BLZ-211 cells using end-to-end PCR (right panel). (B), The full-length cDNA sequence of UCA1a gene. Bold letters, the EST sequence. Red letters, the reverse complement sequence of 5'-RACE GSP1, orange letters, the sequence of 3'-RACE GSP2. Blue letters, the sequence of 5'-end primer and the reverse complement sequence of 3'-end primer. (C), Exon analysis of the UCA1a(CUDR) and UCA1 genes. The UCA1a(CUDR) gene was mapped to human chromosome 19p13.12 positive strand with three exons. (D), Schematic representation of UCA1a(CUDR) and UCA1. Common regions are shown in red.

A

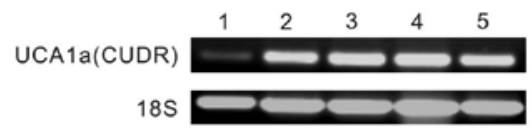

B

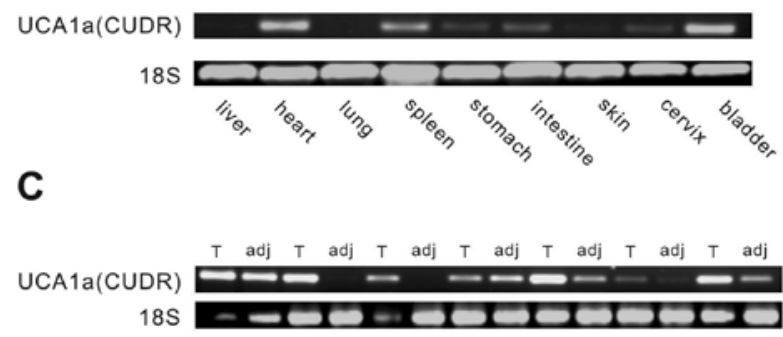

D

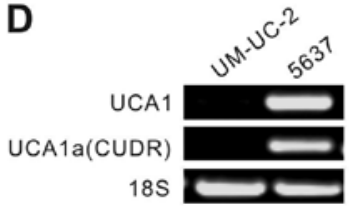

onic development (Fig. 2A). Moreover, UCA1a(CUDR) was upregulated in heart, spleen and bladder, but lower in stomach, intestine and cervix in tissues of 28-week pregnancy (Fig. 2B). The differential expression of UCAla(CUDR) between human bladder TCC and adjacent bladder mucosa was further demonstrated in the tissues from cancer patients. As shown in Fig. 2C, UCA1a(CUDR) was upregulated in bladder cancer tissues (six out of seven) as compared with respective adjacent bladder mucosa tissue counterparts. Furthermore, UCA1a(CUDR) and UCA1 were detected in human bladder cancer 5637 cells but not in UM-UC-2 cells (Fig. 2D). These results suggested that UCA1a(CUDR), in addition to its role in embryonic development, also may be important in the carcinogenesis of bladder cancer.

UCAla(CUDR) overexpression enhances proliferation, migration and invasion of bladder cancer cells in vitro. To gain

Figure 2. The expression patterns of UCA1a(CUDR) gene. (A), UCA1a(CUDR) expression in human normal embryonic tissue. Lanes 1-5, the gestational weeks were three, five, ten, seven, eight, respectively. (B), UCA1a(CUDR) expression in tissues of 28-week pregnancy. (C), UCA1a(CUDR) expression in cDNA pairs of matched human bladder TCC (T) and adjacent bladder mucosa (adj). (D), UCA1a(CUDR) and UCA1 expression in human cancer cell lines. 
A

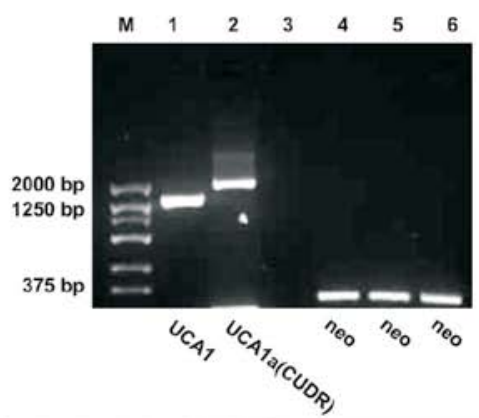

C

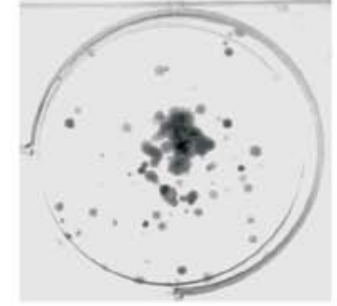

Mock

D

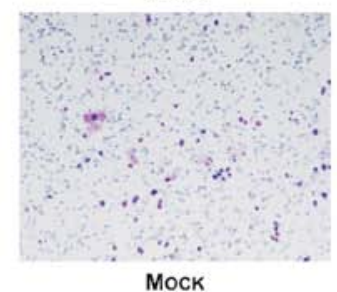

$\mathrm{E}$

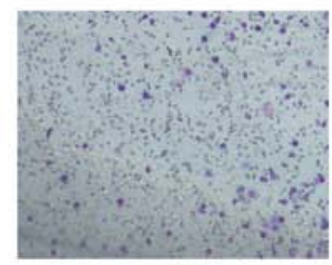

Mock

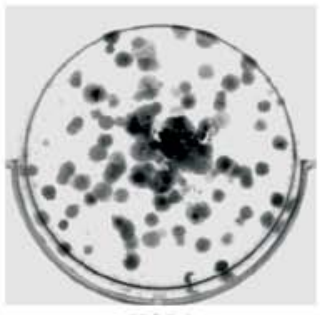

UCA1

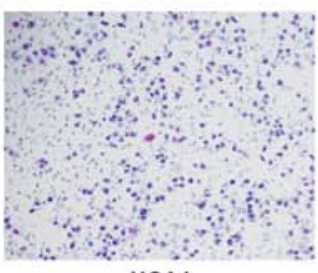

UCA1

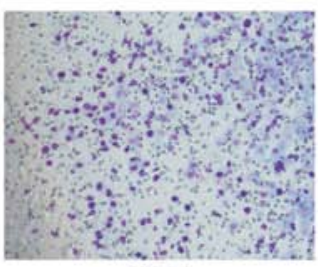

UCA1
B
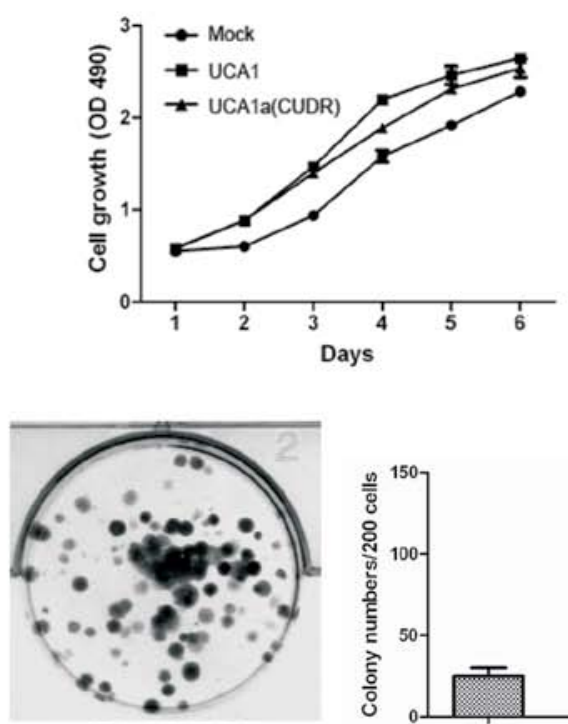

UCA1a(CUDR)

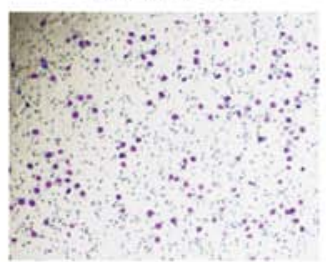

UCA1a(CUDR)

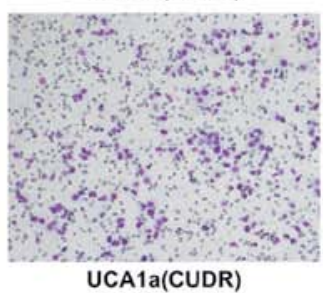

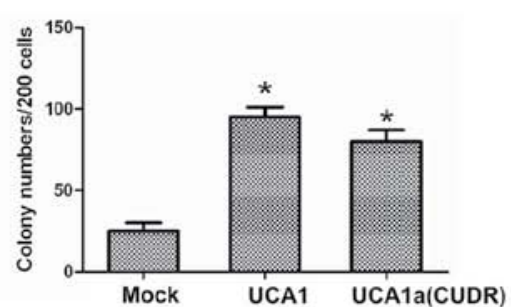
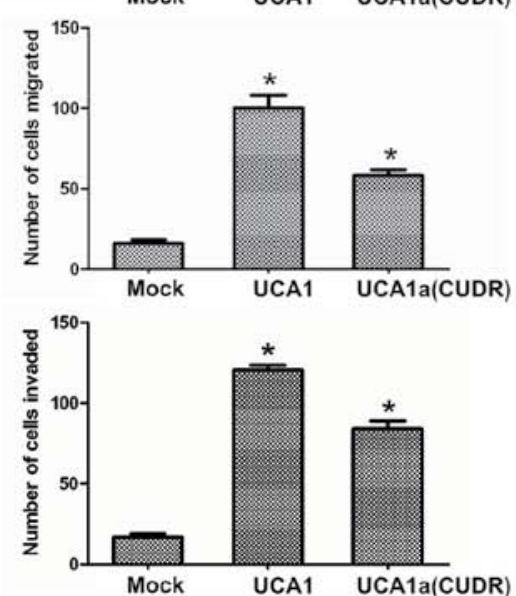

Figure 3. UCA1a(CUDR) overexpression enhances cell proliferation, migration and invasion. UM-UC-2 cells was transfected with pcDNA3.1, UCA1 and UCA1a(CUDR), respectively. (A), RT-PCR analysis showed increased expression of UCA1 or UCA1a(CUDR) in the transfected UM-UC-2 cells. Neo was used as a control. Lanes 1 and 4, UCA1 overexpressing cells; lanes 2 and 5, UCA1a(CUDR) overexpressing cells; lanes 3 and 6, pcDNA3.1 transfected cell. (B), Overexpression of UCA1a(CUDR) significantly promoted UM-UC-2 cell proliferation as measured by MTT assay. The promotion effect became obvious from day 3 , and the difference was significant $(\mathrm{p}<0.05)$. (C), Overexpression of UCA1a(CUDR) increased the clonogenic ability of UM-UC-2 cells $(\mathrm{p}<0.05)$. Photographs of colonies are shown (left panel). The colony numbers were counted and the data are presented as colony numbers per 200 cells. (D, E), Overexpression of UCA1a(CUDR) enhanced cell motility and invasion. Cells were counted in 10 random fields (x200) and presented as the average number of cells per field of view. Values are mean number of migrated cells \pm standard error of the mean. ${ }^{*}$ Significantly different from control $(\mathrm{p}<0.05)$.

insight into the functional role of UCAla(CUDR), a bladder cancer cell line UM-UC-2 was transfected with pcDNA3.1, UCA1 and UCA1a(CUDR), respectively, and overexpression was confirmed by RT-PCR (Fig. 3A). Cells were then analyzed for changes in cell proliferation, migration and invasion. As shown in Fig. 3B, proliferation was significantly increased at $72 \mathrm{~h}$ in UCA1 or UCA1a(CUDR) overexpressing cells when compared with pcDNA3.1 transfected cells. Moreover, colony formation assay showed that, at 14 days after transfection, the plating efficiency for UCA1a(CUDR) overexpressing cells was significantly increased 3.2-fold compared to that for the pcDNA3.1 transfected cells (Fig. 3C). Furthermore, overexpression of UCA1a(CUDR) also significantly increased cell migration (Fig. 3D) and invasion (Fig. 3E), as compared with control, although, the biological effect of UCA1a(CUDR) overexpression was slightly lower than that of UCA1 overexpression. These results indicated that UCA1a(CUDR) enhances proliferation, migration, and invasion of bladder cancer cells in vitro.
UCAla $(C U D R)$ overexpression alters the gene expression profile of $U M-U C-2$. Non-coding RNA is one of the major mechanisms of post-transcriptional regulation of gene expression. To identify genes and pathways that are mediated by UCA1a(CUDR), we next performed DNA microarray analysis and compared gene expression profiles among three transfected UM-UC-2 cells. Hierarchical clustering based on informative probe sets showed that gene expression profile in UCA1a(CUDR) overexpression cells was similar to that of UCA1 overexpression cells, but different from the profile in control cells (Fig. 4A), implying overexpression of UCA1a(CUDR) or UCA1 regulated the gene expression. Careful gene pathway analysis showed that a set of genes had more than 2-fold upregulation or downregulation, and those genes were thought to be involved in tumorigenesis or cell apoptosis (Tables II and III). To validate the results from DNA microarray analysis, the examples of upregulated PDGFB in tumorigenesis pathway and downregulated FAS and ATM in cell apoptosis pathway were 
A
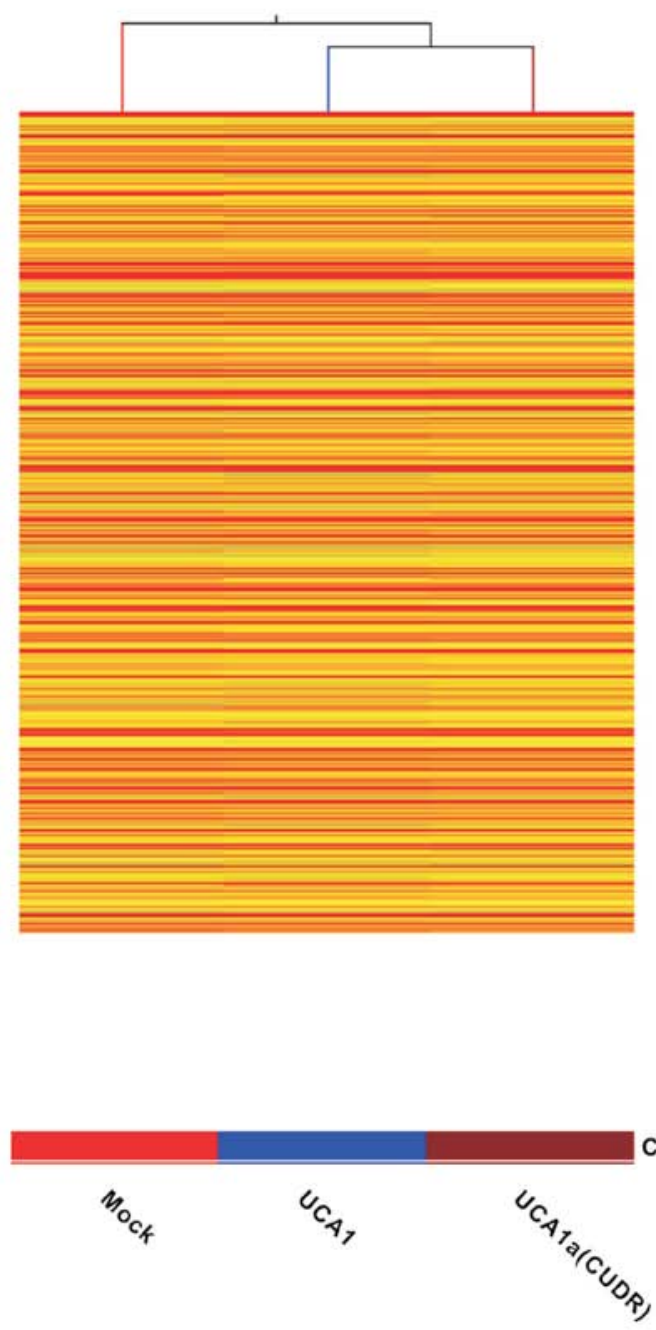

B
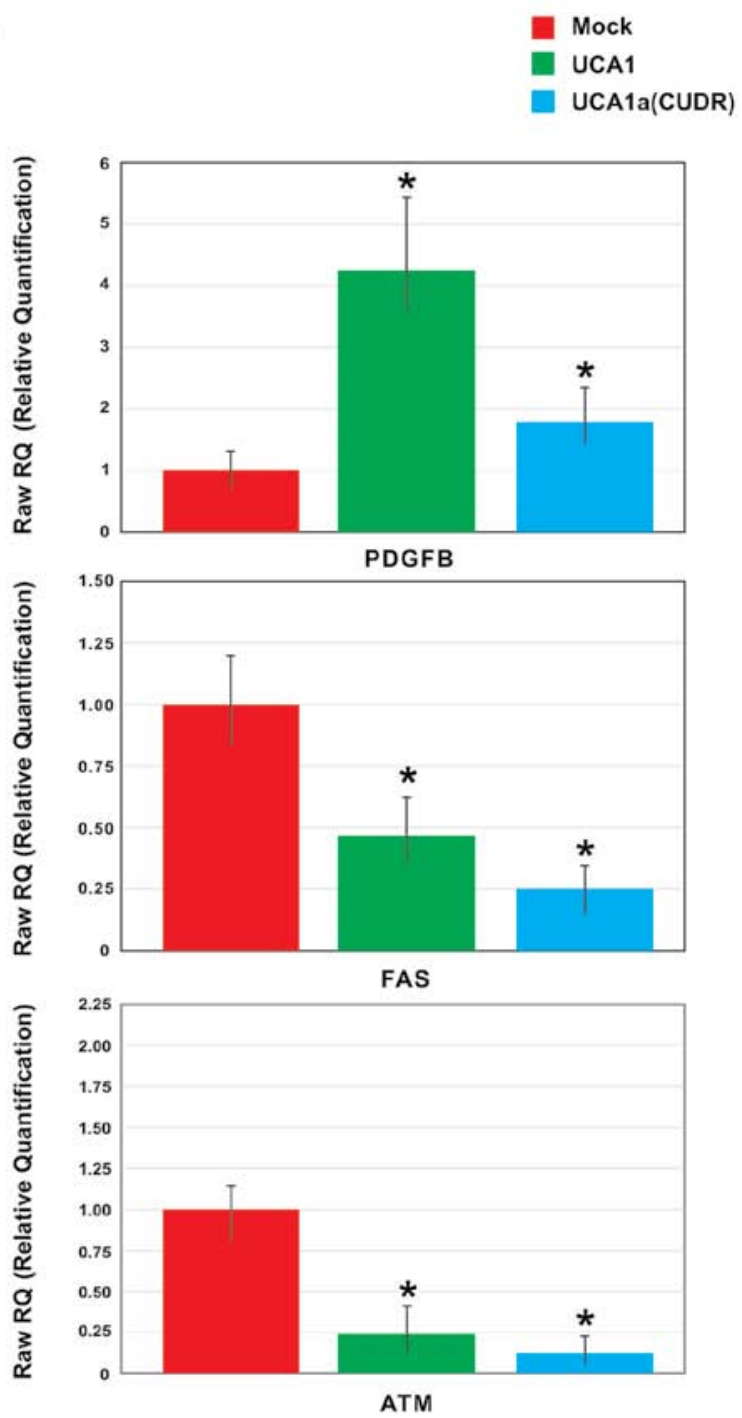

Figure 4. UCA1a(CUDR) overexpression alters the gene expression profile of UM-UC-2. (A), Hierarchical clustering analysis of gene expression profiles in cells transfected with UCA1, UCA1a(CUDR) or pcDNA3.1 empty vector. Columns represent the 41,000+ probe sets detected in all samples. (B), qPCR analysis of mRNA expression of Fas, ATM and PDGFB was performed in three transfected UM-UC-2 cells. Results were normalized to internal control GAPDH mRNA and are presented as relative expressions to mRNA levels in the pcDNA3.1 transfected cells. Data are means $\pm \mathrm{SD}(\mathrm{n}=3)$. "Significantly different from control $(\mathrm{p}<0.05)$.

Table II. Genes with more than 2-fold up-regulation upon UCA1a(CUDR) overexpression.

\begin{tabular}{llll}
\hline GenBank Accession no. & Gene name & \multicolumn{1}{c}{ Description } & Functional category \\
\hline NM_003468 & FZD5 & Frizzled homolog 5 & Pathways in cancer \\
NM_003392 & WNT5A & Wingless-type MMTV integration site family, member 5A & Pathways in cancer \\
NM_014862 & ARNT2 & Aryl-hydrocarbon receptor nuclear translocator 2 & Pathways in cancer \\
NM_002608 & PDGFB & $\begin{array}{l}\text { Platelet-derived growth factor beta polypeptide } \\
\text { [simian sarcoma viral (v-sis) oncogene homolog] }\end{array}$ & Pathways in cancer \\
NM_198129 & LAMA3 & Laminin, alpha 3 (LAMA3), transcript variant 1 & Pathways in cancer \\
\hline
\end{tabular}

further examined by qPCR. The results showed that the mRNA expression level of PDGFB was significantly higher $(\mathrm{p}<0.05)$ in both UCA1a(CUDR) and UCA1 overexpressing cells than in the pcDNA3.1 transfected cells. In contrast, Fas and ATM were significantly $(\mathrm{p}<0.05)$ downregulated in UCA1a(CUDR) and UCA1 overexpressing cells (Fig. 4B). These results suggested that the overexpression of UCA1a(CUDR) or UCA1 might play key roles in cell apoptosis and tumorigenesis pathway, and Fas, 
Table III. Genes with more than 2-fold down-regulation upon UCA1a(CUDR) overexpression.

GenBank Accession no. Gene name

Description

Functional category

\begin{tabular}{|c|c|c|c|}
\hline NM_138957 & MAPK1 & Mitogen-activated protein kinase 1 (MAPK1), transcript variant 2 & Pathways in cancer \\
\hline NM_000963 & PTGS2 & $\begin{array}{l}\text { Prostaglandin-endoperoxide synthase } 2 \\
\text { (prostaglandin G/H synthase and cyclooxygenase) }\end{array}$ & Pathways in cancer \\
\hline AF211169 & FGF1 & Acid fibroblast growth factor-like protein (GLIO703) mRNA & Pathways in cancer \\
\hline NM_212482 & FN1 & Fibronectin 1 & Pathways in cancer \\
\hline NM_005207 & CRKL & v-crk sarcoma virus CT10 oncogene homolog (avian)-like (CRKL) & Pathways in cancer \\
\hline NM_016169 & SUFU & Suppressor of fused homolog (Drosophila) (SUFU) & Pathways in cancer \\
\hline NM_004327 & BCR & Breakpoint cluster region & Pathways in cancer \\
\hline NM_001430 & EPAS 1 & Endothelial PAS domain protein 1 & Pathways in cancer \\
\hline NM_000051 & ATM & Ataxia telangiectasia mutated & Apoptosis \\
\hline
\end{tabular}

$\mathrm{BC} 002763$

PRKAR2A Protein kinase, cAMP-dependent, regulatory, type II, alpha, mRNA Apoptosis (cDNA clone MGC:3606 IMAGE:3629579)

NM_005465 AKT3 V-akt murine thymoma viral oncogene homolog $3 \quad$ Apoptosis

NM_000043 FAS $\quad$ Fas (TNF receptor superfamily, member 6) (FAS) Apoptosis

A

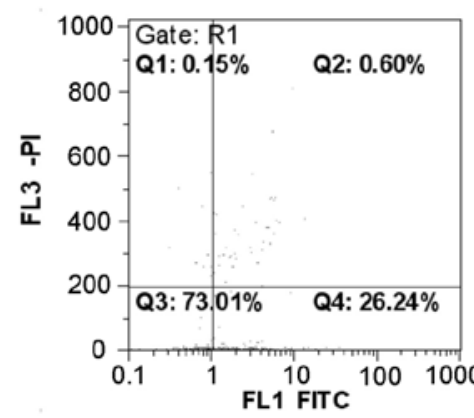

Mock

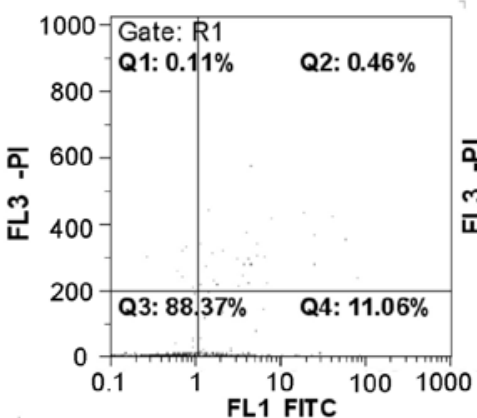

UCA1

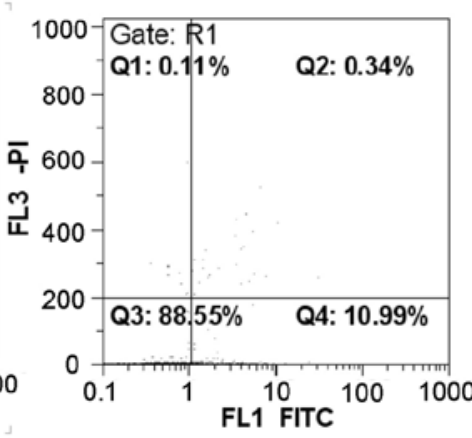

UCA1a(CUDR)

B
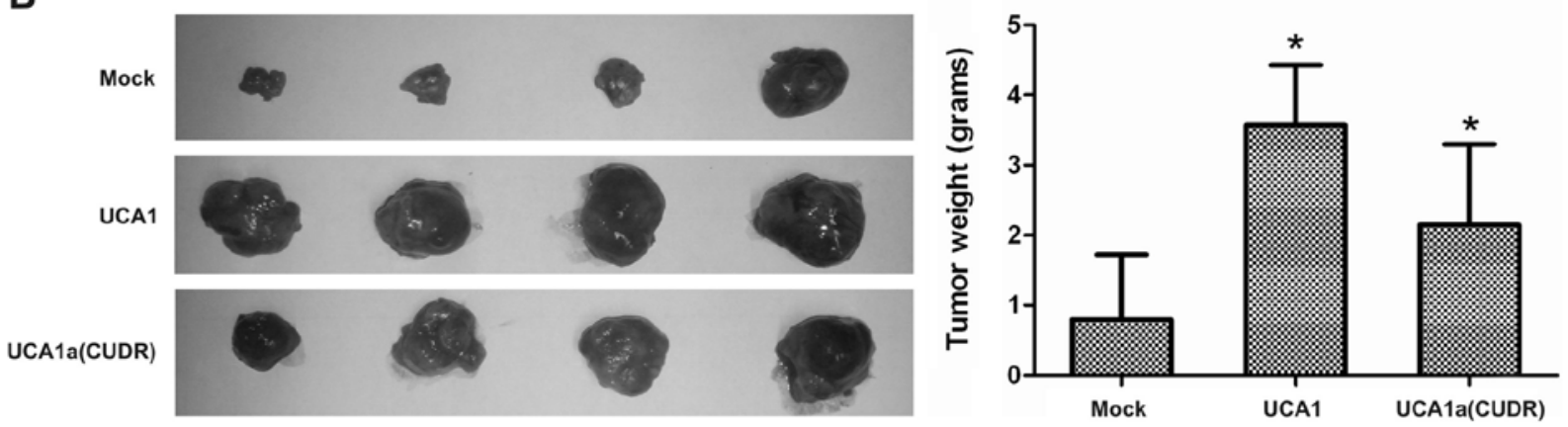

Figure 5. UCA1a(CUDR) overexpression inhibits cell apoptosis and promotes the tumorigenicity of UM-UC-2 cells in vivo. (A), Cisplatin-induced apoptosis in UM-UC-2 cells assessed by Annexin V/propidium iodide staining followed by flow cytometric analysis. Cells were stained with Annexin V/PI after drug treatments for $48 \mathrm{~h}$, and then analyzed for early apoptotic cells (bottom right quadrant) and late apoptotic or dead cells (top right quadrant). The percentages of cells in the four quadrants are shown. Plots are representative of three independent experiments. (B), UCAla(CUDR) overexpression promoted the tumorigenic potential of UM-UC-2 cells in vivo. Photographs of tumor masses excised from the three groups $(\mathrm{n}=4)$ are shown (left panel). The four different tumors in a row are tumors developed in 4 different animals. The mean weight of tumors in UCA1a(CUDR) or UCA1 overexpressing groups was significantly larger than that in control groups. Data are means \pm SD. "Significantly different from control $(\mathrm{p}<0.05)$. 
ATM and PDGFB were regulatory molecules of these signaling pathways, thereby possibly contributing to tumorigenesis.

UCAla(CUDR) overexpression inhibits cell apoptosis and promotes the tumorigenicity of UM-UC-2 cells in vivo. To determine whether UCA1a(CUDR) was associated with cell apoptosis and tumorigenicity in vivo, we first examined the effects of UCA1a(CUDR) on resistance to cisplatin-induced apoptosis in UM-UC-2 cells. By flow cytometric analysis of cells stained with Annexin V/PI, fewer apoptotic cells were detected in UCA1a(CUDR) or UCA1 overexpression cells than in pcDNA3.1 transfected cells after cisplatin treatment (Fig. 5A), indicating that overexpression of UCA1a(CUDR) or UCA1 could antagonize cell apoptosis induced by cisplatin. Next, we examined the effects of UCAla(CUDR) in the tumorigenicity and development of three transfected UM-UC-2 cells were injected subcutaneously into athymic nude mice. As shown in Fig. 5B, the mean weight of tumors in UCA1a(CUDR) or UCA1 overexpressing groups was significantly larger than that in control groups $(\mathrm{p}<0.05)$, suggesting overexpression of UCA1a(CUDR) or UCA1 significantly promoted the tumorigenicity of UM-UC-2 cells in vivo.

\section{Discussion}

Recent genome-wide studies have shown that the mammalian genome is abundantly transcribed, and that at least $80 \%$ of this transcription is exclusively associated with lncRNAs $(12,13)$. Emerging data strongly implicate lncRNAs in the basal regulation of protein-coding genes, which are central to normal development and oncogenesis, at both the transcriptional and the posttranscriptional levels (3). In this study, we successfully assembled a new full-length cDNA of UCAla using homology search in EST database of the NCBI and confirmed its validity by experiment. By genomic analysis, the sequence of UCA1a was identical to that of CUDR, which indicated that they were actually the same gene. Previous results have demonstrated that CUDR might exert its function as an lncRNA in the regulation of drug resistance and cellular transformation (10). In addition, overexpression of CUDR has also been reported in various human tumors, including those cancers originating from colon, cervix, and lung (10). Here, we demonstrated that UCAla(CUDR) might play an important role in bladder cancer. Our data showed that the expression patterns of UCAla(CUDR) was similar to that of UCA1 (8), indicated that it was also an embryonic development and bladder cancer-associated gene. Especially, overexpression of UCA1a(CUDR) was able to enhance proliferation, tumorigenesis, as well as invasiveness of UM-UC-2 cells, suggesting that UCA1a(CUDR) may exert its function in the regulation of bladder cancer development, growth, and invasion.

The way that IncRNA regulates the expression of its target genes is not clear. The RNA might interact with its binding proteins and then through some still unidentified pathways regulate the expression of target genes. In microarray assays, we demonstrated that UCA1a(CUDR) overexpression cells have alterations in signaling pathways regulating cell apoptosis and tumorigenesis and identified several genes. Among them, PDGFB is well documented to be associated with the development of cancer (14), while Fas and ATM are known for regulating cell apoptosis (15-17). Refractoriness to apoptosis is believed to be one of the possible causes of cancer formation. This may explain why some of the genes in the apoptotic pathway have been shown to be associated with the development of cancer (18). Therefore, we speculated that UCA1a(CUDR) promoted the tumorigenicity of UM-UC-2 cells mediated through these potential target genes. Future efforts will be devoted to understanding the underlying molecular mechanism by which UCA1a(CUDR) up or downregulates these target genes.

It is of great interest that UCA1a(CUDR) and UCA1 have similar trends of expression and functions in bladder cancer, indicating that they might be regulated by a common transcriptional factor. Of note, some important transcription factors, such as p53, NF- $\kappa$ B, Sox2, Oct4 and Nanog, have been found to be involved in transcriptional regulation of other lncRNAs (19). Therefore, searching for regulator to both UCA1a(CUDR) and UCA1 is critical for better understanding of the biological mechanism of lncRNA.

In 2006, another transcript of Evf-1 gene generated by alternative splicing was reported, which was denominated Evf-2. Sequence comparisons of the Evf-1 and Evf-2 cDNA sequences reveal unique 5'- and 3'-ends and a 2,561-bp common region. Another interesting finding is that the Evf-2 5'-region may be necessary and sufficient for activity (20). Similarly, our results revealed that UCA1a(CUDR) and UCA1 cDNA sequences incorporated a 1,265-bp common region (Fig. 1D). More importantly, we found that the biological effect of UCA1a(CUDR) overexpression was milder than that of UCA1 overexpression, including in vitro and in vivo. Given both the significant similarities and differences between UCA1 and UCA1a(CUDR), we propose that the common region may be critical for their biological activity and the additional sequence on the 3'-ends of UCA1a(CUDR) may decrease the mRNA level. Therefore, understanding what part of the sequence of the common region contributes to observed activity may be important to design more specific and effective molecular therapies.

In summary, we show that UCAla(CUDR) is another transcript of UCA1 gene. Both UCA1 and UCA1a(CUDR) have similar functions in modulating the growth and tumorigenesis of bladder cancer, and their common region may be critical for the biological activity. Therefore, this study extends our knowledge on the oncogenesis of bladder cancer, and indicates that the common region between UCA1 and UCA1a(CUDR) may serve as a new molecular target for bladder cancer therapy.

\section{Acknowledgements}

This work was supported by a grant from the National Natural Science Foundation of China (no. 30973429).

\section{References}

1. Mallardo M, Poltronieri P and D'Urso OF: Non-protein coding RNA biomarkers and differential expression in cancers: a review. J Exp Clin Cancer Res 27: 19, 2008.

2. Umlauf D, Fraser P and Nagano T: The role of long non-coding RNAs in chromatin structure and gene regulation: variations on a theme. Biol Chem 389: 323-331, 2008.

3. Mercer TR, Dinger ME and Mattick JS: Long non-coding RNAs: insights into functions. Nat Rev Genet 10: 155-159, 2009.

4. Morris KV and Vogt PK: Long antisense non-coding RNAs and their role in transcription and oncogenesis. Cell Cycle 9: 2544-2547, 2010. 
5. Gibb EA, Brown CJ and Lam WL: The functional role of long noncoding RNA in human carcinomas. Mol Cancer 10: 38, 2011.

6. Huarte $M$ and Rinn JL: Large non-coding RNAs: missing links in cancer? Hum Mol Genet 19: R152-R161, 2010.

7. Yang YC, Li X and Chen W: Screening and identification of heterogeneous phenotype-associated genes in human bladder cancer. Nan Fang Yi Ke Da Xue Xue Bao 26: 270-274, 2006.

8. Wang F, Li X, Xie X, Zhao L and Chen W: UCA1, a non-proteincoding RNA up-regulated in bladder carcinoma and embryo, influencing cell growth and promoting invasion. FEBS Lett 582 1919-1927, 2008.

9. Wang XS, Zhang Z, Wang HC, et al: Rapid identification of UCA1 as a very sensitive and specific unique marker for human bladder carcinoma. Clin Cancer Res 12: 4851-4858, 2006.

10. Tsang WP, Wong TW, Cheung AH, Co CN and Kwok TT: Induction of drug resistance and transformation in human cancer cells by the noncoding RNA CUDR. RNA 13: 890-898, 2007.

11. Huang X and Madan A: Cap3: a DNA sequence assembly program. Genome Res 9: 868-877, 1999.

12. Birney E, Stamatoyannopoulos JA, Dutta A, et al: Identification and analysis of functional elements in $1 \%$ of the human genome by the ENCODE pilot project. Nature 447: 799-816, 2007.

13. Amit NK and Paul MH: Mining mammalian transcript data for functional long non-coding RNÂs. PLoS One 5: e10316, 2010.
14. Kang DW and Min do S: Platelet derived growth factor increases phospholipase D1 but not phospholipase D2 expression via NFkappaB signaling pathway and enhances invasion of breast cancer cells. Cancer Lett 294: 125-133, 2010.

15. Houston A and O'Connell J: The Fas signalling pathway and its role in the pathogenesis of cancer. Curr Opin Pharmacol 4: 321-326, 2004.

16. Greiner TC, Dasgupta C, Ho VV, et al: Mutation and genomic deletion status of ataxia telangiectasia mutated (ATM) and p53 confer specific gene expression profiles in mantle cell lymphoma. Proc Natl Acad Sci USA 103: 2352-2357, 2006.

17. Mitui M, Nahas SA, Du LT, et al: Functional and computational assessment of missense variants in the ataxia-telangiectasia mutated (ATM) gene: mutations with increased cancer risk. Hum Mutat 30: $12-21,2009$.

18. Moscat J and Diaz-Meco MT: p62 at the crossroads of autophagy, apoptosis, and cancer. Cell 137: 1001-1004, 2009.

19. Guttman M, Amit I, Garber M, et al: Chromatin signature reveals over a thousand highly conserved large non-coding RNAs in mammals. Nature 458: 223-227, 2009.

20. Feng J, Bi C, Clark BS, Mady R, Shah P and Kohtz JD: The Evf-2 noncoding RNA is transcribed from the Dlx-5/6 ultraconserved region and functions as a Dlx-2 transcriptional coactivator. Genes Dev 20: 1470-1484, 2006. 